\title{
TENSOR PRODUCTS OF CERTAIN BANACH MODULES OVER COMMUTATIVE CONVOLUTION MEASURE ALGEBRAS
}

\author{
KARI YLINEN
}

\section{Introduction and preliminaries}

J. E. Kerlin [6] has studied Banach algebra actions of one group algebra $L^{1}(G)$ of a locally compact abelian topological group $G$ on another such algebra. In particular, Kerlin was concerned with locally compact abelian groups $K, G_{1}, \ldots, G_{n}$, Banach algebra actions of $L^{1}(K)$ on $L^{1}\left(G_{1}\right), \ldots, L^{1}\left(G_{n}\right)$, and the $L^{1}(K)$-tensor product $L^{1}\left(G_{1}\right) \otimes_{L^{1}(K)} \ldots$ $\otimes_{L^{1}(K)} L^{1}\left(G_{n}\right)$ in the sense of M. A. Rieffel [10] of the resulting Banach $L^{1}(K)$-modules. The objects of study in the present paper are related to those of Kerlin. The Banach algebras we consider, namely the commutative convolution measure algebras introduced by J. L. Taylor in [15], are much more general than the group algebras $L^{1}(G)$. A convolution measure algebra is a complex $L$-space with a Banach algebra product which is suitably related to the $L$-space structure. The class of convolution measure algebras includes e.g. the convolution algebra $M(S)$ of bounded regular Borel measures on any locally compact topological semigroup $S$ with a separately continuous multiplication (see [16, Theorem 2.1]), and the so-called $L$-subalgebras [16, p. 812] of $M(S)$. But it appears that in the context of this rather general class of Banach algebras one needs more restrictions on the algebra actions than those assumed by Kerlin in the case of group algebra modules. Specifically, we introduce what we shall call a CCM-action of one commutative convolution measure algebra on another. This notion generalizes naturally the multiplication in a commutative convolution measure algebra. Before making this statement precise we review some aspects of the theory of normed tensor products and convolution measure algebras.

If $E$ and $F$ are Banach spaces, $E \hat{\otimes} F$ denotes their projective tensor product (i.e. the completion of the algebraic tensor product $E \otimes F$ with 
respect to the greatest cross-norm $\gamma$ of Schatten [13, p. 38]) and $E \hat{\otimes} F$ the completion (called the weak tensor product of $E$ and $F$ in [14, p. 355]) of $E \otimes F$ with respect to the least one, $\lambda$, of all cross-norms whose dual norms are also cross-norms (see $[13$, p. 32]). In this paper the $\lambda$-norm appears in tensor products of spaces $C\left(X_{i}\right)$ of continuous complex functions on compact Hausdorff spaces $X_{i}, i=1,2$. In this connection the crucial property is the natural identification $C\left(X_{1} \times X_{2}\right)=C\left(X_{1}\right) \hat{\otimes} C\left(X_{2}\right)$ $[14$, p. 357]. The $\gamma$-norm will be used mainly in tensor products of complex $L$-spaces. We take as our definition the characterization of a complex $L$-space as the predual (see $\left[12\right.$, p. 1]) of a commutative $W^{*}$-algebra as in [19]. More precisely, a complex $L$-space $M$ is an ordered Banach space whose dual, with the order determined by that of $M$, is a commutative $C^{*}$-algebra. We often suppress the word "complex", because all Banach spaces we consider are over the complex field. If $M$ and $N$ are $L$-spaces, $M \hat{\otimes} N$ is an $L$-space whose positive cone is the closed convex hull of the set $\{\mu \otimes v \mid \mu \in M, \mu \geq 0, v \in N, \nu \geq 0\}$, and whose dual can be identified with the $W^{*}$-tensor product $M^{*} \bar{\otimes} N^{*}$. For details see e.g. $[17$, pp. $20-22]$ and $[19$, pp. 5-6]. In general the properties of tensor products of Banach spaces and Banach algebras we use without explicit mention are elementary and can be found in [19] and the references listed there. In particular, the projective tensor product of two Banach algebras will be regarded as a Banach algebra with the product described in [19, p. 4].

If $M$ is a complex $L$-space, the $W^{*}$-algebra structure in $M^{*}$ compatible with the norm and order determined by $M$ is by the BanachStone theorem unique. A bounded linear map $T: M \rightarrow N$, where $M$ and $N$ are complex $L$-spaces, is called an $L$-homomorphism, if its transpose $T^{*}: N^{*} \rightarrow M^{*}$ is what we shall call a $C_{1}^{*}$-morphism, i.e. a $C^{*}$-algebra homomorphism which maps the identity of $N^{*}$ to that of $M^{*}$. A convolution measure algebra (abbreviated as CM-algebra in the sequel) is a complex $L$-space $M$ with a Banach algebra product $(\mu, v) \mapsto \mu v$ such that the bounded linear map $\Theta: M \widehat{\otimes} M \rightarrow M$ for which $\Theta(\mu \otimes v)=\mu \nu$, $\mu, v \in M$, is an $L$-homomorphism. In this paper we limit our attention to commutative CM-algebras. A central position in their theory is occupied by the structure semigroup constructed by J. L. Taylor in [15]. We shall not go into details in this introductory section, but refer the reader e.g. to [17] for an exposition.

The Banach modules we are considering in this paper generalize commutative CM-algebras in the following sense. If $C$ and $A$ are commutative CM-algebras and $\Theta: C \widehat{\otimes} A \rightarrow A$ is an $L$-homomorphism, we say that $\Theta$ is a CCM-action and $A$ is a CCM-module over $C$, provided that 
the bilinear map from $C \times A$ to $A$ defined by $\Theta$ satisfies the natural algebraic conditions postulated in [6, p. 3], i.e. $\Theta\left(\left(c c^{\prime}\right) \otimes a\right)=$ $\Theta\left(c \otimes \Theta\left(c^{\prime} \otimes a\right)\right)$ and $\Theta\left(c \otimes\left(a a^{\prime}\right)\right)=\Theta(c \otimes a) a^{\prime}=a \Theta\left(c \otimes a^{\prime}\right), \quad c, c^{\prime} \in C$, $a, a^{\prime} \in A$.

The main results of Section 3 concern the $C$-tensor product $A \otimes_{C} B$ (in the sense of [10] and [6]) of two commutative CM-algebras $A$ and $B$ which are CCM-modules over a commutative CM-algebra $C$. It is shown that $A \otimes_{C} B$ is also a commutative CM-algebra (Theorem 3.1). In Section 4 the structure semigroup of $A \otimes_{C} B$ is given a characterization (Theorem 4.2) which generalizes Theorem 4.3 in [19]. Section 5 concerns mainly group algebras which are CCM-modules over group algebras. Specifically, it is shown that if $K$ and $G$ are locally compact abelian topological groups and $\Theta: L^{1}(K) \hat{\otimes} L^{1}(G) \rightarrow L^{1}(G)$ is a CCM-action, then $\Theta\left(L^{1}(K) \widehat{\otimes} L^{1}(G)\right)=$ $L^{1}(G)$ if and only if there is a continuous homomorphism $\varphi: K \rightarrow G$ such that $\Theta(\nu \otimes \mu)=\widetilde{\varphi}(v) * \mu, v \in L^{1}(K), \mu \in L^{1}(G)$, where $\widetilde{\varphi}: M(K) \rightarrow M(G)$ is the algebra homomorphism canonically induced by $\varphi$.

Notations. For any commutative (complex) Banach algebra $A, \Delta(A)$ denotes the spectrum of $A$, i.e. the set of nonzero multiplicative linear functionals on $A$ equipped with the relative weak* topology. If $E$ is a Banach space and $D \subset E$, then $[D]^{-}$is the closed linear span of $D$ in $E$, and $D^{0}$ is the polar of $D$ in the topological dual $E^{*}$ of $E$. The identity map of a set $D$ is denoted by $i d_{D}$.

\section{Banach $C$-algebras and their $C$-tensor products}

Throughout this section $C$ denotes a commutative Banach algebra. We adopt the following definition used by J. E. Kerlin (see [6, p. 3]).

Definition 2.1. A commutative Banach algebra $A$ is a commutative Banach $C$-algebra with respect to a bounded bilinear map $(c, a) \mapsto c \cdot a$ from $C \times A$ to $A$ if

$$
\begin{gathered}
\left(c c^{\prime}\right) \cdot a=c \cdot\left(c^{\prime} \cdot a\right) \text { and } \\
c \cdot\left(a a^{\prime}\right)=(c \cdot a) a^{\prime}=a\left(c \cdot a^{\prime}\right)
\end{gathered}
$$

for all $c, c^{\prime} \in C$ and $a, a^{\prime} \in A$.

By abuse of language we shall call a bounded linear map $\Theta: C \widehat{\otimes} A \rightarrow A$ a commutative Banach algebra module action (of $C$ on $A$ ), abbreviated as CBAM-action, if $A$ is a commutative Banach $C$-algebra with respect to the bilinear map $(c, a) \mapsto c \cdot a$ for which $c \cdot a=\Theta(c \otimes a), c \in C, a \in A$. We continue to use the notation $\Theta(c \otimes a)=c \cdot a$ for every CBAM-action $\Theta$. This should cause no confusion. 
Suppose $\Theta_{A}: C \hat{\otimes} A \rightarrow A$ and $\Theta_{B}: C \hat{\otimes} B \rightarrow B$ are CBAM-actions. Modelling on M. A. Rieffel's definition (see [10]) of a tensor product of Banach modules, J. E. Kerlin defined in [6] the $C$-tensor product of $A$ and $B$ as follows. Denote

$$
J=[\{(c \cdot a) \otimes b-a \otimes(c \cdot b) \mid c \in C, a \in A, b \in B\}]^{-C A} \hat{\otimes} B .
$$

As observed in [6, p. 5], $J$ is a closed ideal in the commutative Banach algebra $A \hat{\otimes} B$, so that the quotient algebra $A \otimes_{C} B=A \hat{\otimes} B / J$ with the quotient norm is a commutative Banach algebra, called the $C$-tensor product of $A$ and $B$. We denote $a \otimes_{C} b=\pi(a \otimes b)$, where $\pi: A \hat{\otimes} B \rightarrow$ $A \hat{\otimes} B / J$ is the quotient map, so that $A \otimes_{C} B=\left[\left\{a \otimes_{C} b \mid a \in A\right.\right.$, $b \in B\}]^{-}$.

Two natural CBAM-actions of $C$ on $A \hat{\otimes} B$ arise from $\Theta_{A}$ and $\Theta_{B}$. Indeed, if $j: C \hat{\otimes}(A \hat{\otimes} B) \rightarrow(C \hat{\otimes} A) \hat{\otimes} B$ and $k: C \hat{\otimes}(A \hat{\otimes} B) \rightarrow$ $A \hat{\otimes}(C \hat{\otimes} B)$ denote the canonical isometric isomorphisms satisfying $j(c \otimes(a \otimes b))=(c \otimes a) \otimes b$ and $k(c \otimes(a \otimes b))=a \otimes(c \otimes b), \quad$ it is easy to verify that $\Theta_{1}=\left(\Theta_{A} \widehat{\otimes} i d_{B}\right) \circ j$ (resp. $\Theta_{2}=\left(i d_{A} \widehat{\otimes} \Theta_{B}\right) \circ k$ ) is a CBAMaction of $C$ on $A \hat{\otimes} B$ such that $c \cdot(a \otimes b)=(c \cdot a) \otimes b$ (resp. $c \cdot(a \otimes b)=$ $a \otimes(c \cdot b))$. Applying the commutativity of $C$ and (i) in Definition 2.1 we see that for each $c \in C$ the kernel of each one of the operators $x \mapsto \pi \circ \Theta_{1}(c \otimes x)$ and $x \mapsto \pi \circ \Theta_{2}(c \otimes x)$ from $A \hat{\otimes} B$ to $A \hat{\otimes} B / J$ contains $J$. Both of these operators induce canonically the same operator $T_{c}: A \hat{\otimes} B / J \rightarrow A \hat{\otimes} B / J$, and the operator $\Theta: C \widehat{\otimes}\left(A \otimes_{C} B\right) \rightarrow A \otimes_{C} B$ for which $\Theta(c \otimes x)=T_{c} x, \quad x \in A \otimes_{C} B$, is a CBAM-action of $C$ on $A \otimes_{C} B$ such that $c \cdot\left(a \otimes_{C} b\right)=(c \cdot a) \otimes_{C} b \quad\left(=a \otimes_{C}(c \cdot b)\right)$. We shall always regard $A \otimes_{C} B$ as a commutative Banach $C$-algebra with respect to the action so defined.

We close this section with an alternate description of the multiplication in the $C$-tensor product of two Banach $C$-algebras.

$\mathrm{L} \mathrm{e} \mathrm{m} \mathrm{m} \mathrm{a} \mathrm{2.1.} \mathrm{Let} A$ and $B$ be commutative Banach $C$-algebras. There is a unique isometric isomorphism

$$
\varphi:\left(A \otimes_{C} B\right) \otimes_{C}\left(A \otimes_{C} B\right) \rightarrow\left(A \otimes_{C} A\right) \otimes_{C}\left(B \otimes_{C} B\right)
$$

which maps $\left(a_{1} \otimes_{C} b_{1}\right) \otimes_{C}\left(a_{2} \otimes_{C} b_{2}\right)$ to $\left(a_{1} \otimes_{C} a_{2}\right) \otimes_{C}\left(b_{1} \otimes_{C} b_{2}\right)$ for $a_{1}, a_{2} \in A$, $b_{1}, b_{2} \in B$. Let $m_{A}: A \hat{\otimes} A \rightarrow A$ and $m_{B}: B \widehat{\otimes} B \rightarrow B$ denote the multiplication maps of $A$ and $B$, and write

$$
\begin{aligned}
& J_{A}=\left[\left\{\left(c \cdot a_{1}\right) \otimes a_{2}-a_{1} \otimes\left(c \cdot a_{2}\right) \mid c \in C, a_{1}, a_{2} \in A\right\}\right]^{-}, \\
& J_{B}=\left[\left\{\left(c \cdot b_{1}\right) \otimes b_{2}-b_{1} \otimes\left(c \cdot b_{2}\right) \mid c \in C, b_{1}, b_{2} \in B\right\}\right]^{-} .
\end{aligned}
$$


Then $m_{A} \mid J_{A}=0$ and $m_{B} \mid J_{B}=0$ so that $m_{A}$ and $m_{B}$ induce canonically the maps $\tilde{m}_{A}: A \hat{\otimes} A / J_{A} \rightarrow A$ and $\tilde{m}_{B}: B \hat{\otimes} B / J_{B} \rightarrow B$ for which $\tilde{m}_{A}\left(x+J_{A}\right)=m_{A}(x), \quad \tilde{m}_{B}\left(x+J_{B}\right)=m_{B}(x)$. There is a unique bounded linear map

$$
\tilde{m}_{A} \otimes_{C} \tilde{m}_{B}:\left(A \otimes_{C} A\right) \otimes_{C}\left(B \otimes_{C} B\right) \rightarrow A \otimes_{C} B
$$

such that $\tilde{m}_{A} \otimes_{C} \tilde{m}_{B}\left(x \otimes_{C} y\right)=\tilde{m}_{A}(x) \otimes_{C} \tilde{m}_{B}(y), x \in A \otimes_{C} A, y \in B \otimes_{C} B$. If $\pi:\left(A \otimes_{C} B\right) \hat{\otimes}\left(A \otimes_{C} B\right) \rightarrow\left(A \otimes_{C} B\right) \otimes_{C}\left(A \otimes_{C} B\right)$ denotes the natural quotient map, then $\left(\tilde{m}_{A} \otimes_{C} \tilde{m}_{B}\right) \circ \varphi \circ \pi$ is the linear operator corresponding to the multiplication in $A \otimes_{C} B$.

Proof. Since $A, B, A \otimes_{C} A, B \otimes_{C} B, A \otimes_{C} B$ and $B \otimes_{C} A$ are $C$ - $C$-bimodules in the sense of $[10$, p. 447], the existence of $\varphi$ can be proved by applying Theorem 2.11 and Corollary 2.6 in [10]. The uniqueness of $\varphi$ is clear. Condition (ii) in Definition 2.1 shows that $m_{A} \mid J_{A}=0$ and $m_{B} \mid J_{B}=0$. The existence and uniqueness of $\widetilde{m}_{A} \otimes_{C} \widetilde{m}_{B}$ follow from Theorem 2.7 in [10], since by Definition 2.1 (ii) $\tilde{m}_{A} \in \operatorname{Hom}_{C}\left(A \otimes_{C} A, A\right)$ and $\widetilde{m}_{B} \in \operatorname{Hom}_{C}\left(B \otimes_{C} B, B\right)$ in the notation of [10]. Suppose $a_{1}, a_{2} \in A$, $b_{1}, b_{2} \in B$. The multiplication in the quotient algebra $A \otimes_{C} B$ gives by definition $\left(a_{1} \otimes_{C} b_{1}\right)\left(a_{2} \otimes_{C} b_{2}\right)=a_{1} a_{2} \otimes_{C} b_{1} b_{2}$. The last statement is thus clear, since

$$
\left(\left(\tilde{m}_{A} \otimes_{C} \tilde{m}_{B}\right) \circ \varphi \circ \pi\right)\left(\left(a_{1} \otimes_{C} b_{1}\right) \otimes\left(a_{2} \otimes_{C} b_{2}\right)\right)=a_{1} a_{2} \otimes_{C} b_{1} b_{2} .
$$

\section{CCM-modules and their tensor products}

As indicated in the introductory section, this paper is mainly concerned with a special class of CBAM-actions. These are defined as follows.

Definition 3.1. Let $C$ and $A$ be commutative CM-algebras. If the CBAM-action $\Theta: C \hat{\otimes} A \rightarrow A$ is an $L$-homomorphism, we say that $\Theta$ is a CCM-action and $A$ is a CCM-module over $C$ (with respect to the action $\Theta$ ).

Before the main result of this section (Theorem 3.1) we prove four lemmas.

$\mathrm{L}$ e $\mathrm{m} \mathrm{m}$ a 3.1. Let $M$ be a complex $L$-space and $J$ a closed subspace of $M$ such that $J^{0}$ is a sub- $C^{*}$-algebra of $M^{*}$ containing its identity. Then $M / J$ is a complex L-space and the quotient map $\pi: M \rightarrow M / J$ is an L-homomorphism, if $M / J$ is equipped with the quotient norm and the following order: $[x]=x+J \geq 0$ if and only if $\langle x, f\rangle \geq 0$ for all $f \geq 0$ in $J^{0}$. If $N$ is another $L$-space and $T: M \rightarrow N$ an L-homomorphism whose kernel contains $J$, then the induced map $\widetilde{T}: M / J \rightarrow N$, for which $\widetilde{T}[x]=T x$, is an L-homomorphism. 
Proof. Since $\pi^{*}:(M / J)^{*} \rightarrow M^{*}$ is an isometry whose range is $J^{0}$, $(M / J)^{*}$ has the structure of a $C^{*}$-algebra with identity such that $\pi^{*}$ is a $C_{1}^{*}$-morphism. Clearly, the order determined by $(M / J)^{*}$ in $M / J$ can be described as stated. As to the second statement, observe that since $\pi^{*} \circ \widetilde{T}^{*}=T^{*}$ is a $C_{1}^{*}$-morphism and $\pi^{*}$ is an injective $C_{1}^{*}$-morphism, $\widetilde{T}^{*}$ is a $C_{1}^{*}$-morphism.

In [7, p. 663] the order in $M / J$ defined in the above lemma is called the order induced by $J^{0}$. The definition is clearly independent of the choice of the representative in $[x] \in M / J$. We shall refer to this order as the quotient order in $M / J$. This term is justified by the following lemma.

$\mathrm{L}$ e $\mathrm{m} \mathrm{m}$ a 3.2. Let $M$ and $J$ be as in Lemma 3.1. The positive cone of $M / J$ with the order defined in Lemma 3.1 is the image of the positive cone of $M$ under the quotient map $\pi: M \rightarrow M / J$.

Proof. It is immediate that $\pi(\mu) \geq 0$ for every $\mu \geq 0$ in $M$. Suppose now $\mu \in M$ is such that $\pi(\mu) \geq 0$. We show that in $M$ there is $v \geq 0$ for which $\pi(v)=\pi(\mu)$. Let us consider $M^{*}$ realized as a von Neumann algebra of operators on a Hilbert space $H$ so that the $\sigma\left(M^{*}, M\right)$-topology on $M^{*}$ corresponds to the relative $\sigma$-weak operator topology (see [12, pp. 39-42], [2, pp. 38-40]). Then $J^{0}$ is identified with a von Neumann algebra of operators on $H$, and $\mu \mid J^{0}$ is a $\sigma$-weakly continuous positive linear functional on $J^{0}$. Thus there is a sequence $\left(\xi_{n}\right)_{n=1}^{\infty}$ in $H$ such that $\sum_{n=1}^{\infty}\left\|\xi_{n}\right\|^{2}<\infty$ and $\langle x, \mu\rangle=\sum_{n=1}^{\infty}\left(x \xi_{n}, \xi_{n}\right), \quad x \in J^{0}$ [2, p. 51]. Using this expression $\mu \mid J^{0}$ can be extended to a $\sigma$-weakly continuous positive linear functional $v$ on $M^{*}$. As $J$ is $\sigma\left(M, M^{*}\right)$-closed, the polar of $J^{0}$ in $M$ is $J$ by the bipolar theorem, so that $\pi(v)=\pi(\mu)$, and the lemma is proved.

$\mathrm{L} \mathrm{e} \mathrm{m} \mathrm{m}$ a 3.3. Let $C, A$ and $B$ be complex L-spaces and $\varphi: C \hat{\otimes} A \rightarrow A$, $\psi: C \hat{\otimes} B \rightarrow B$ L-homomorphisms. Denote $\varphi(c \otimes a)=c \cdot a, \quad \psi(c \otimes b)=$ $c \cdot b$, and

$$
J=[\{(c \cdot a) \otimes b-a \otimes(c \cdot b) \mid c \in C, a \in A, b \in B\}]^{-C A} \hat{\otimes} B .
$$

Let $\pi: A \hat{\otimes} B \rightarrow A \hat{\otimes} B / J$ be the quotient map. Then $J^{0}$ is a sub- $C^{*}$ algebra of $(A \hat{\otimes} B)^{*}$ containing its identity. Equipped with the quotient order $A \hat{\otimes} B / J$ is a complex $L$-space whose positive cone is the closed convex hull of $\{\pi(a \otimes b) \mid a \geq 0, a \in A, b \geq 0, b \in B\}$, and $\pi$ is an $L$ homomorphism.

Proof. From the definition of the positive cone in the projective tensor product of two $L$-spaces (Section 1) it follows that the canonical isometric isomorphism $j: C \hat{\otimes}(A \hat{\otimes} B) \rightarrow(C \hat{\otimes} A) \hat{\otimes} B$ is also an order isomorphism. Therefore $T_{1}=\left(\varphi \hat{\otimes} i d_{B}\right) \circ j$ is an $L$-homomorphism from $C \hat{\otimes}(A \hat{\otimes} B)$ 
to $A \hat{\otimes} B \quad$ such that $T_{1}(c \otimes(a \otimes b))=(c \cdot a) \otimes b \quad[19$, Theorem 3.2]. Similarly, we get an $L$-homomorphism $T_{2}: C \hat{\otimes}(A \hat{\otimes} B) \rightarrow A \hat{\otimes} B$ such that $T_{2}(c \otimes(a \otimes b))=a \otimes(c \cdot b)$. Since $\left\{f \in(A \widehat{\otimes} B)^{*} \mid T_{1}^{*} f=T_{2}^{*} f\right\}=$ $\left\{f \in(A \hat{\otimes} B)^{*} \mid f((c \cdot a) \otimes b-a \otimes(c \cdot b))=0\right.$ for all $c \in C, a \in A$, $b \in B\}=J^{0}$, and $T_{1}^{*}$ and $T_{2}^{*}$ are $C_{1}^{*}$-morphisms, $J^{0}$ is a sub- $C^{*}$ algebra of $(A \hat{\otimes} B)^{*}$ containing its identity. The last statement in the lemma is a consequence of the first part, Lemmas 3.1 and 3.2, and the definition of the positive cone in $A \hat{\otimes} B$.

Convention. Whenever $C, A, B, \varphi, \psi$, and $J$ are as in the above lemma, $A \hat{\otimes} B / J$ will be regarded as an $L$-space with the structure described in the lemma. In particular, if $C, A$ and $B$ are commutative CM-algebras and $\Theta_{A}: C \hat{\otimes} A \rightarrow A, \Theta_{B}: C \hat{\otimes} B \rightarrow B$ are CCM-actions, then $A \otimes_{C} B$ is an $L$-space.

$\mathrm{L}$ e $\mathrm{m} \mathrm{m}$ a 3.4. Let $C, A_{j}, B_{j}$ be complex L-spaces and $\varphi_{j}: C \widehat{\otimes} A_{j} \rightarrow$ $A_{j}, \quad \psi_{j}: C \hat{\otimes} B_{j} \rightarrow B_{j} \quad$ L-homomorphisms, $j=1,2 . \quad$ Denote $J_{j}=$ $\left[\left\{\varphi_{j}(c \otimes a) \otimes b-a \otimes \psi_{j}(c \otimes b) \mid c \in C, \quad a \in A_{j}, \quad b \in B_{j}\right\}\right]^{-} \subset A_{j} \widehat{\otimes} B_{j}$, and let $\pi_{j}: A_{j} \hat{\otimes} B_{j} \rightarrow A_{j} \hat{\otimes} B_{j} / J_{j}$ be the quotient map. If $\alpha: A_{1} \rightarrow A_{2}$ and $\beta: B_{1} \rightarrow B_{2}$ are L-homomorphisms such that $\alpha \varphi_{1}(c \otimes a)=\varphi_{2}(c \otimes \alpha a)$ and $\beta \psi_{1}(c \otimes b)=\psi_{2}(c \otimes \beta b), \quad c \in C, \quad a \in A_{1}, \quad b \in B_{1}$, then there is a unique bounded linear map $\alpha \otimes_{C} \beta: A_{1} \hat{\otimes} B_{1} / J_{1} \rightarrow A_{2} \hat{\otimes} B_{2} / J_{2}$ for which $\left(\alpha \otimes_{C} \beta\right) \pi_{1}(a \otimes b)=\pi_{2}(\alpha a \otimes \beta b), \quad a \in A_{1}, \quad b \in B_{1}, \quad$ and $\quad \alpha \otimes_{C} \beta$ is an L-homomorphism.

Proof. The existence and uniqueness of $\alpha \otimes_{C} \beta$ follow from Theorem 2.7 in $[10$, p. 450$]$, the crucial point being the fact that by our assumptions the kernel of $\pi_{2} \circ(\alpha \hat{\otimes} \beta)$ contains $J_{1}$. Since $\pi_{2} \circ(\alpha \hat{\otimes} \beta)$ is an $L$-homomorphism by Theorem 3.2 in [19] and Lemma 3.3, an application of Lemmas 3.3 and 3.1 completes the proof, $\alpha \otimes_{C} \beta$ being simply the operator canonically induced by $\pi_{2} \circ(\alpha \hat{\otimes} \beta)$ on the quotient space $A_{1} \widehat{\otimes} B_{1} / J_{1}$.

Th e or e m 3.1. Let $C, A$ and $B$ be commutative CM-algebras and $\Theta_{A}: C \hat{\otimes} A \rightarrow A, \Theta_{B}: C \hat{\otimes} B \rightarrow B$ CCM-actions. Then $A \otimes_{C} B$ is a commutative CM-algebra and the bounded linear map $\Theta: C \widehat{\otimes}\left(A \otimes_{C} B\right) \rightarrow A \otimes_{C} B$ for which $\Theta\left(c \otimes\left(a \otimes_{C} b\right)\right)=(c \cdot a) \otimes_{C} b=a \otimes_{C}(c \cdot b) \quad$ (see the discussion preceding Lemma 2.1) is a CCM-action.

Proof. As observed before Lemma 3.4, $A \otimes_{C} B$ is an $L$-space. To show that it is a CM-algebra we use Lemma 2.1, which gives a factorization

$$
\begin{gathered}
\left(A \otimes_{C} B\right) \hat{\otimes}\left(A \otimes_{C} B\right) \stackrel{\pi}{\rightarrow}\left(A \otimes_{C} B\right) \otimes_{C}\left(A \otimes_{C} B\right) \stackrel{\varphi}{\rightarrow} \\
\left(A \otimes_{C} A\right) \otimes_{C}\left(B \otimes_{C} B\right) \stackrel{\tilde{m}_{A} \otimes_{C} \tilde{m}_{B}}{\longrightarrow} A \otimes_{C} B
\end{gathered}
$$


of the multiplication map of $A \otimes_{C} B$ (the notations being as in Lemma 2.1). We shall prove that $\pi, \varphi$ and $\widetilde{m}_{A} \otimes_{C} \widetilde{m}_{B}$ are $L$-homomorphisms. Let us first show that the CBAM-action $\Theta: C \widehat{\otimes}\left(A \otimes_{C} B\right) \rightarrow A \otimes_{C} B$ is an $L$ homomorphism. Let $j: C \hat{\otimes}(A \hat{\otimes} B) \rightarrow(C \hat{\otimes} A) \hat{\otimes} B$ be the isometric isomorphism satisfying $j(c \otimes(a \otimes b))=(c \otimes a) \otimes b \quad$ and $\quad \pi_{0}: A \widehat{\otimes} B \rightarrow$ $A \otimes_{C} B$ the natural quotient map. The diagram

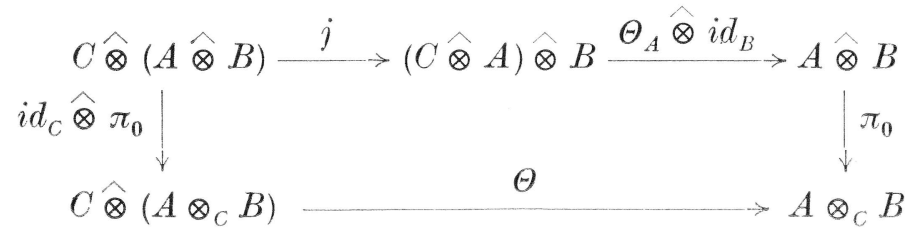

is commutative. By the definition of the positive cone in the projective tensor product of two $L$-spaces $j$ is also an order isomorphism. Since $\pi_{0}$ is an $L$-homomorphism (Lemma 3.3), and $i d_{C} \widehat{\otimes} \pi_{0}$ and $\Theta_{A} \widehat{\otimes} i d_{B}$ are $L$-homomorphisms by Theorem 3.2 in [19], $\left(\pi_{0} \circ\left(\Theta_{A} \widehat{\otimes} i d_{B}\right) \circ j\right)^{*}$ is a $C_{1}^{*}$-morphism and $\left(\mathrm{id}_{C} \hat{\otimes} \pi_{0}\right)^{*} \quad$ is an injective $C_{1}^{*}$-morphism. As $\left(i d_{C} \hat{\otimes} \pi_{0}\right)^{*} \circ \Theta^{*}=\left(\pi_{0} \circ\left(\Theta_{A} \hat{\otimes} i d_{B}\right) \circ j\right)^{*}, \quad \Theta^{*}$ is thus a $C_{1}^{*}$-morphism, i.e. $\Theta$ is an $L$-homomorphism. Replacing here $B$ by $A$ (resp. $A$ by $B$ ) we see that the CBAM-actions $\Theta_{1}: C \hat{\otimes}\left(A \otimes_{C} A\right) \rightarrow A \otimes_{C} A$ and $\Theta_{2}: C \hat{\otimes}\left(B \otimes_{C} B\right) \rightarrow B \otimes_{C} B$ for which $\Theta_{1}\left(c \otimes\left(a_{1} \otimes_{C} a_{2}\right)\right)=\left(c \cdot a_{1}\right) \otimes_{C} a_{2}$ and $\Theta_{2}\left(c \otimes\left(b_{1} \otimes_{C} b_{2}\right)\right)=\left(c \cdot b_{1}\right) \otimes b_{2}, c \in C, a_{1}, a_{2} \in A, b_{1}, b_{2} \in B$, are $L$-homomorphisms. Lemma 3.3 now shows that $\pi$ is an $L$-homomorphism and the isometry $\varphi$ is an order isomorphism. As $\left(J_{A}\right)^{0}$ (in the notation of Lemma 2.1) is by Lemma 3.3 a sub- $C^{*}$-algebra of $(A \widehat{\otimes} A)^{*}$ containing its identity, $\widetilde{m}_{A}: A \otimes_{C} A \rightarrow A$ is an $L$-homomorphism by Lemma 3.1. Similarly, $\tilde{m}_{B}$ is an $L$-homomorphism, and so is $\widetilde{m}_{A} \otimes_{C} \widetilde{m}_{B}$ by Lemma 3.4 . Thus $A \otimes_{C} B$ is by definition a commutative CM-algebra, and $\Theta$ is a CCM-action.

\section{The structure semigroup of $A \otimes_{C} B$}

Throughout this section $C, A$ and $B$ are commutative CM-algebras and $\Theta_{A}: C \hat{\otimes} A \rightarrow A, \quad \Theta_{B}: C \hat{\otimes} B \rightarrow B$ are CCM-actions. We write $P_{C}=[\Delta(C)]^{-} C C^{*}$. As $\Delta(C)$ is closed with respect to multiplication and involution, $P_{C}$ is a sub- $C^{*}$-algebra of $C^{*}$ containing its identity, so that $P_{C}$ can be identified via the Gelfand transformation with $C\left(S_{C}\right)$, the $C^{*}$-algebra of continuous complex functions on $S_{C}$, where $S_{C}=$ $\Delta\left(P_{C}\right) . S_{C}$ is a compact abelian topological semigroup with a multiplication 
$(x, y) \mapsto x y$ which is determined by the condition $x y(\gamma)=x(\gamma) y(\gamma)$, $\gamma \in \Delta(C)$. Equipped with this structure, $S_{C}$ is called the structure semigroup of $S_{C}$. The Gelfand transformation puts $\Delta(C)$ in a bijective correspondence with $\widehat{S_{C}}$, the set of the (nonzero continuous) semicharacters on $S_{C}$. The canonical embedding $c \mapsto \widetilde{c}$ of $C$ into $M\left(S_{C}\right),\langle c, f\rangle=\langle f, \widetilde{c}\rangle$ for $f \in P_{C}=C\left(S_{C}\right)$, where $M\left(S_{C}\right)=C\left(S_{C}\right)^{*}$ is the Banach space of bounded regular Borel measures on $S_{C}$, is an algebra homomorphism with respect to the convolution product

$$
\mu * v(f)=\int_{S_{C}} \int_{S_{C}} f(x y) d \mu(x) d v(y), f \in C\left(S_{C}\right),
$$

in $M\left(S_{C}\right)$. Similar notations and terminology are used when $C$ is replaced by $A$ or $B$. The details of the theory summarized above can be found e.g. in [15, pp. $157-159]$ and $[17$, pp. $24-25]$, see also $[19$, pp. $7-8]$ and [20, Theorem 2.1].

In [19] we have shown that the structure semigroup of the CM-algebra $A \hat{\otimes} B$ can be identified with the product $S_{A} \times S_{B}$. The main purpose of this section is to prove an analogue of that result for $A \otimes_{C} B$ instead of $A \hat{\otimes} B$ (Theorem 4.2). Certain identifications and notational conventions will be adopted. For example, $P_{C} \widehat{\otimes} P_{A}$ is sometimes identified with $C\left(S_{C} \times S_{A}\right)$ and sometimes with a closed subspace of $C^{*} \hat{\otimes} A^{*}$ (see [13, p. 35]), which in turn is considered canonically and isometrically (see $\left[13\right.$, p. 40] ) embedded in $(C \widehat{\otimes} A)^{*}=C^{*} \bar{\otimes} A^{*}$. In general our approach is the same as in [19]. A fundamental result of Gelbaum [4] and Tomiyama [18] yields the identification $\Delta(C \widehat{\otimes} A)=\{\varphi \otimes \psi \mid \varphi \in \Delta(C), \psi \in \Delta(A)\}$, and as in the proof of Theorem 4.3 in [19] it is seen that $P_{C} \widehat{\otimes} P_{A}=$ $[\Delta(C \hat{\otimes} A)]^{-}$. Since $\Theta_{A}\left(\left(c_{1} \otimes a_{1}\right)\left(c_{2} \otimes a_{2}\right)\right)=\Theta_{A}\left(c_{1} c_{2} \otimes a_{1} a_{2}\right)=\left(c_{1} c_{2}\right)$. $\left(a_{1} a_{2}\right)=c_{1} \cdot\left[c_{2} \cdot\left(a_{2} a_{1}\right)\right]=c_{1} \cdot\left[\left(c_{2} \cdot a_{2}\right) a_{1}\right]=\left(c_{1} \cdot a_{1}\right)\left(c_{2} \cdot a_{2}\right)=$ $\Theta_{A}\left(c_{1} \otimes a_{1}\right) \Theta_{A}\left(c_{2} \otimes a_{2}\right), c_{1}, c_{2} \in C, a_{1}, a_{2} \in A$, and $C \hat{\otimes} A=[\{c \otimes a\}$ $c \in C, a \in A\}]^{-}, \Theta_{A}$ is an algebra homomorphism. Therefore $\Theta_{A}^{*}\left(P_{A}\right) \subset$ $P_{C} \hat{\otimes} P_{A}$. We let $T_{A}: P_{A} \rightarrow P_{C} \hat{\otimes} P_{A}$, i.e. $T_{A}: C\left(S_{A}\right) \rightarrow C\left(S_{C} \times S_{A}\right)$, denote the map defined by $\Theta_{A}^{*}$ restricted to $P_{A}$. In much of the discussion below attention is limited to $\Theta_{A}$, but we use the corresponding results and notations for $\Theta_{B}$ as well.

$\mathrm{L} \mathrm{e} \mathrm{m} \mathrm{m}$ a 4.1. There is a unique mapping $t_{A}: \Delta(A) \rightarrow \Delta(C) \cup\{0\}$ (called by abuse of language the adjoint map of the action $\Theta_{A}$, compare [6, p. 11]) such that

$$
\left\langle c, t_{A}(\gamma)\right\rangle\langle a, \gamma\rangle=\langle c \cdot a, \gamma\rangle, \quad c \in C, \quad a \in A, \quad \gamma \in \Delta(A) .
$$


The mapping $t_{A}$ is weak* continuous, and

$$
\Theta_{A}^{*} \gamma=t_{A}(\gamma) \otimes \gamma, \gamma \in \Delta(A) \text {. }
$$

Proof. The existence and weak* continuity of $t_{A}$ are shown in $[6$, Lemma 3.1]. The uniqueness of $t_{A}$ is clear, for if $\langle a, \gamma\rangle \neq 0$, then $\left\langle t_{A}(\gamma), c\right\rangle=\langle\gamma, a\rangle^{-1}\langle\gamma, c \cdot a\rangle$. If $c \in C, \quad a \in A$ and $\gamma \in \Delta(A)$, then $\left\langle c \otimes a, t_{A}(\gamma) \otimes \gamma\right\rangle=\left\langle c, t_{A}(\gamma)\right\rangle\langle a, \gamma\rangle=\langle c \cdot a, \gamma\rangle=\left\langle c \otimes a, \Theta_{A}^{*} \gamma\right\rangle$, so that (1) holds.

Since the second conjugate space of a commutative $C^{*}$-algebra is a commutative $W^{*}$-algebra (see $\left.[12, \mathrm{p} .43],[3, \mathrm{p} .236]\right), M\left(S_{C}\right)=C\left(S_{C}\right)^{*}$, $M\left(S_{A}\right)$ and $M\left(S_{C} \times S_{A}\right)$ are by definition complex $L$-spaces (and the order in each space as a predual is the same as the order in a space of measures $\left[3\right.$, p. 236]). There is a linear isometry $j_{A}: M\left(S_{C}\right) \hat{\otimes} M\left(S_{A}\right) \rightarrow M\left(S_{C} \times S_{A}\right)$ which maps $\mu \otimes v$ to the product measure $\mu \times v$ for $\mu \in M\left(S_{C}\right), v \in M\left(S_{A}\right)$. This can be proved using the techniques of the proof of Theorem 2.2 in [8]. The same proof shows that $j_{A}\left(M\left(S_{C}\right) \otimes M\left(S_{A}\right)\right)=\left\{m \in M\left(S_{C} \times S_{A}\right)\right\}$ $m \ll \mu \times v$ for some $\mu \geq 0$ in $M\left(S_{C}\right), \quad v \geq 0$ in $\left.M\left(S_{A}\right)\right\}$ (« denotes absolute continuity), and applying the Radon-Nikodym theorem it can be proved that the positive cone of $j_{A}\left(M\left(S_{C}\right) \hat{\otimes} M\left(S_{A}\right)\right)$ is the closed convex hull of $\left\{\mu \times v \mid \mu \in M\left(S_{C}\right), \quad \mu \geq 0, \quad v \in M\left(S_{A}\right), \quad v \geq 0\right\}$. Therefore $j_{A} x \geq 0$ is equivalent to $x \geq 0, x \in M\left(S_{C}\right) \widehat{\otimes} M\left(S_{A}\right)$. It is also clear that $j_{A}\left(M\left(S_{C}\right) \hat{\otimes} M\left(S_{A}\right)\right)$ is an $L$-subspace of $M\left(S_{C} \times S_{A}\right)$ in the sense of $[15, \mathrm{p} .151]$. It is not difficult to prove, using the fact that $\left(j_{A}\left(M\left(S_{C}\right) \hat{\otimes} M\left(S_{A}\right)\right)\right)^{0}$ is a * -ideal in $C\left(S_{C} \times S_{A}\right)^{* *}$ (see Theorems 3.1 and 3.2 in [7]), that $j_{A}^{*}$ is a $C_{1}^{*}$-morphism. But instead of going into the details of that proof we observe that $j_{A}$ satisfies the conditions of Definition 1.3 in [15, p. 152], and so $j_{A}$ is an $L$-homomorphism in the sense of the definition we are using in this paper (cf. [15, Theorem 1.2] or [7, Theorem $4.2])$.

We are going to show that $M\left(S_{A}\right)$ is a CCM-module over $M\left(S_{C}\right)$ with respect to a suitable action. This action $\Phi_{A}: M\left(S_{C}\right) \hat{\otimes} M\left(S_{A}\right) \rightarrow M\left(S_{A}\right)$ is defined as $\Phi_{A}=T_{A}^{*} \circ j_{A}$. We denote also $\Phi_{A}(\mu \otimes v)=\mu \cdot v$.

$\mathrm{L}$ e $\mathrm{m} \mathrm{m}$ a 4.2. If $t_{A}$ is the adjoint map of the action $\Theta_{A}$, then $\langle\mu \cdot v, \gamma\rangle=\left\langle\mu, t_{A}(\gamma)\right\rangle\langle v, \gamma\rangle$ for all $\mu \in M\left(S_{C}\right), v \in M\left(S_{A}\right), \gamma \in \Delta(A)=$ $\widehat{S}_{A}$.

Proof. Using Lemma 4.1 we get $\langle\mu \cdot v, \gamma\rangle=\left\langle\mu \times v, \Theta_{A}^{*} \gamma\right\rangle=\langle\mu \times v$, $\left.t_{A}(\gamma) \otimes \gamma\right\rangle=\left\langle\mu, t_{A}(\gamma)\right\rangle\langle\nu, \gamma\rangle \quad$ with the interpretation $t_{A}(\gamma) \otimes \gamma \in$ $C\left(S_{C} \times S_{A}\right)$.

Theorem 4.1. The mapping $\Phi_{A}: M\left(S_{C}\right) \hat{\otimes} M\left(S_{A}\right) \rightarrow M\left(S_{A}\right)$ is a CCM-action . 
Proof. Let us first note the validity of the algebraic conditions

$$
\begin{aligned}
& \left(\mu * \mu^{\prime}\right) \cdot v=\mu \cdot\left(\mu^{\prime} \cdot v\right) \text { and } \\
& \mu \cdot\left(v * v^{\prime}\right)=(\mu \cdot v) * v^{\prime} \text { for } \mu, \mu^{\prime} \in M\left(S_{C}\right), \quad v, \quad v^{\prime} \in M\left(S_{A}\right) .
\end{aligned}
$$

Applying Lemma 4.2 three times we get

$$
\begin{gathered}
\left\langle\left(\mu * \mu^{\prime}\right) \cdot v, \gamma\right\rangle=\left\langle\mu * \mu^{\prime}, t_{A}(\gamma)\right\rangle\langle v, \gamma\rangle= \\
\left\langle\mu, t_{A}(\gamma)\right\rangle\left\langle\mu^{\prime}, t_{A}(\gamma)\right\rangle\langle v, \gamma\rangle=\left\langle\mu, t_{A}(\gamma)\right\rangle\left\langle\mu^{\prime} \cdot v, \gamma\right\rangle=\left\langle\mu \cdot\left(\mu^{\prime} \cdot v\right), \gamma\right\rangle
\end{gathered}
$$

for all $\gamma \in \widehat{S}_{A}$, and since $C\left(S_{A}\right)=\left[\widehat{S}_{A}\right]^{-}$, (i) holds. An analogous argument yields (ii). As $M\left(S_{C}\right)$ and $M\left(S_{A}\right)$ are known to be CMalgebras (in fact, what we say below gives with the choice $A=C$ this result, too), the theorem is proved once $\Phi_{A}$ is shown to be an $L$ homomorphism. But $T_{A}$ is a $C_{1}^{*}$-morphism, and so is its second transpose when $P_{A}^{* *}$ and $\left(P_{C} \hat{\otimes} P_{A}\right)^{* *}$ are equipped with the canonical structure of the enveloping von Neumann algebra [3, p. 237]. (Note that a Banach space is weak * dense in its bidual, and in the bidual of a $C^{*}$-algebra involution is weak * continuous and multiplication separately weak * continuous). Since $j_{A}$ is an $L$-homomorphism, $\Phi_{A}=T_{A}^{*} \circ j_{A}$ is therefore an $L$-homomorphism.

We now turn to the problem of characterizing the structure semigroup of $A \otimes_{C} B$. The answer is given in Theorem 4.2, which generalizes Theorem 4.3 in [19].

$\mathrm{L}$ e $\mathrm{m} \mathrm{m}$ a 4.3. If $S$ and $T$ are compact Hausdorff spaces and $\mu \in M(S)$, then the map $v \mapsto \mu \times v, v \in M(T)$, is continuous from $\sigma(M(T), C(T))$ to $\sigma(M(S \times T), C(S \times T))$.

Proof. If $f \in C(S \times T)$, the function $\varphi, \varphi(y)=\int_{S} f(x, y) d \mu(x)$, is continuous on $T$. This proves the lemma, because $\langle f, \mu \times v\rangle=\langle\varphi, v\rangle$.

In the next theorem we denote as before

$$
J=[\{(c \cdot a) \otimes b-a \otimes(c \cdot b) \mid c \in C, a \in A, b \in B\}]^{-} \subset A \widehat{\otimes} B .
$$

We embed e.g. $S_{C}$ canonically in $M\left(S_{C}\right)$, and write accordingly $\Phi_{A}\left(\delta_{u} \otimes \delta_{s}\right)=u \cdot s$ for the Dirac measures $\delta_{u}$ and $\delta_{s}$.

Theorem 4.2. For a pair $(\varphi, \psi)$ where $\varphi \in \Delta(A)=\widehat{S}_{A}$ and $\psi \in \Delta(B)=\widehat{S}_{B}$, the following five conditions are equivalent:

(i) $\varphi \otimes \psi \in J^{0}$,

(ii) $t_{A}(\varphi)=t_{B}(\psi)$ (in the notation of Lemma 4.1),

(iii) $\langle c \cdot a, \varphi\rangle\langle b, \psi\rangle=\langle a, \varphi\rangle\langle c \cdot b, \psi\rangle$ for all $c \in C, \quad a \in A, \quad b \in B$, 
(iv) $\varphi(u \cdot s) \psi(t)=\varphi(s) \psi(u \cdot t)$ for all $u \in S_{C}, s \in S_{A}, t \in S_{B}$,

(v) $\langle v \cdot \lambda, \varphi\rangle\langle\mu, \psi\rangle=\langle\lambda, \varphi\rangle\langle v \cdot \mu, \psi\rangle$ for all $v \in M\left(S_{C}\right), \lambda \in M\left(S_{A}\right), \mu \in M\left(S_{B}\right)$.

The set $\Delta=J^{0} \cap \Delta(A \widehat{\otimes} B)$ with its relative weak ${ }^{*}$ topology is homeomorphic to the spectrum of $A \otimes_{C} B=A \hat{\otimes} B / J$ under the map $\gamma \mapsto \gamma \circ \pi$ where $\pi: A \hat{\otimes} B \rightarrow A \hat{\otimes} B / J$ is the quotient map. The closed linear span $P$ of $\triangle$ is a sub-C*-algebra of $P_{A} \widehat{\otimes} P_{B}$ containing its identity. There is a semigroup multiplication $(x, y) \mapsto x y$ in $\Delta(P)$ satisfying $x y(\gamma)=x(\gamma) y(\gamma), \gamma \in \Delta$, and with this product $\Delta(P)$ is topologically isomorphic to the structure semigroup of $A \otimes_{C} B$. There is a continuous surjective semigroup homomorphism $\varkappa$ from the product $S_{A} \times S_{B}$ onto the structure semigroup of $A \otimes_{C} B$ such that $x\left(x_{1}, y_{1}\right)=x\left(x_{2}, y_{2}\right)$ if and only if $\varphi\left(x_{1}\right) \psi\left(y_{1}\right)=\varphi\left(x_{2}\right) \psi\left(y_{2}\right)$ for all pairs $(\varphi, \psi) \in \widehat{S}_{A} \times \widehat{S}_{B}$ satisfying e.g. (iv).

Proof. The equivalence of (i), (ii) and (iii) is contained in the proof of [6, Theorem 3.3], and so is the description of the spectrum of $A \otimes_{C} B$. As the case of two factors is notationally simple to handle, we include for completeness a proof of this part of the theorem, too. From the definition of $J$ it follows that (i) is equivalent to requiring that

$$
\langle\varphi \otimes \psi,(c \cdot a) \otimes b-a \otimes(c \cdot b)\rangle=0 \text { for all } c \in C, a \in A, b \in B,
$$

i.e. to (iii). The formula in (iii) is by Lemma 4.1 equivalent to $\left\langle c, t_{A}(\varphi)\right\rangle\langle a, \varphi\rangle\langle b, \psi\rangle=\langle a, \varphi\rangle\left\langle c, t_{B}(\psi)\right\rangle\langle b, \psi\rangle$, and since $a$ and $b$ can be chosen to make $\langle a, \varphi\rangle$ and $\langle b, \psi\rangle$ nonzero, (ii) and (iii) are equivalent. It follows from Lemmas 4.1 and 4.2 that

$$
\widetilde{c} \cdot a=\widetilde{c} \cdot \widetilde{a}, c \in C, a \in A,
$$

where e.g. $c \mapsto \widetilde{c}$ is the canonical embedding of $C$ in $M\left(S_{C}\right)$. In fact, $\langle\widetilde{c} \cdot \vec{a}, \gamma\rangle=\langle c \cdot a, \gamma\rangle=\left\langle c, t_{A}(\gamma)\right\rangle\langle a, \gamma\rangle=\left\langle\tilde{c}, t_{A}(\gamma)\right\rangle\langle\widetilde{a}, \gamma\rangle=\langle\widetilde{c} \cdot \tilde{a}, \gamma\rangle$ for all $\gamma \in P_{A}=\widehat{S}_{A}$. Thus (v) implies (iii). It is even more obvious that (v) implies (iv). Let us now show that (iii) implies (v). As the canonical image $\widetilde{A}=\{\widetilde{a} \mid a \in A\}$ of $A$ is weak* dense in $M\left(S_{A}\right)$, there is a net $\left(\widetilde{a}_{\alpha}\right)$ in $\widetilde{A}$ converging to $\lambda$ in the weak* topology. By (iii) and (1), $\left\langle\widetilde{c} \cdot \widetilde{a}_{\alpha}, \varphi\right\rangle\langle\tilde{b}, \psi\rangle=\left\langle\widetilde{a}_{\alpha}, \varphi\right\rangle\langle\widetilde{c} \cdot \widetilde{b}, \psi\rangle$. As $T_{A}^{*}$ is continuous from $\sigma\left(M\left(S_{C} \times S_{A}\right), C\left(S_{C} \times S_{A}\right)\right)$ to $\sigma\left(M\left(S_{A}\right), C\left(S_{A}\right)\right)$, it follows from Lemma 4.3 that for fixed $m \in M\left(S_{C}\right)$ the map $h \mapsto m \cdot h=T_{A}^{*}(m \times h)$ from $M\left(S_{A}\right)$ into itself is weak* continuous. Therefore $\langle\widetilde{c} \cdot \lambda, \varphi\rangle\langle\tilde{b}, \psi\rangle=$ $\langle\lambda, \varphi\rangle\langle\tilde{c} \cdot \widetilde{b}, \psi\rangle$. Iterating this argument we obtain $(\mathrm{v})$, when $\mu$ is next approximated by elements from $\widetilde{B}=\{\widetilde{b} \mid b \in B\} \subset M\left(S_{B}\right)$, and finally $v$ 
by elements from $\widetilde{C}$. Since it follows at once from (iv) that (v) holds for linear combinations of Dirac measures, and such measures are weak* dense in the whole space of measures, a similar proof shows that (iv) implies (v). The fact that $\Delta$ and $\Delta\left(A \otimes_{C} B\right)$ are homeomorphic under $\gamma \mapsto \gamma \circ \pi$ follows from Theorem 3.1.17 in [9, p. 116]. By Theorem 3.1 $A \otimes_{C} B$ is a CM-algebra so that $P_{1}=\left[\Delta\left(A \otimes_{C} B\right)\right]^{-}$is a sub-C*-algebra of $\left(A \otimes_{C} B\right)^{*}$ containing its identity. Since $\pi^{*}:\left(A \otimes_{C} B\right)^{*} \rightarrow(A \hat{\otimes} B)^{*}$ is by definition (see Lemmas 3.1 and 3.3) an isometric $C_{1}^{*}$-morphism which maps $\Delta\left(A \otimes_{C} B\right)$ bijectively onto $\Delta, P=[\Delta]^{-}$is a $C^{*}$-algebra with identity (this could easily be proved also directly by using (iv)) and $\pi^{*}$ defines a $C^{*}$-algebra isomorphism of $P_{1}$ onto $P$. It is now clear that $\Delta(P)$ has a semigroup multiplication such that $x y(\gamma)=x(\gamma) y(\gamma), x, y \in \Delta(P), \gamma \in \Delta$, and $\Delta(P)$ is topologically isomorphic to the structure semigroup of $A \otimes_{C} B$. If the structure semigroup $\Delta\left([\Delta(A \hat{\otimes} B)]^{-}\right)$of $A \hat{\otimes} B$ is identified with $S_{A} \times S_{B}$ in accordance with [19, Theorem 4.3], $x$ arises as the dual mapping of $\pi^{*} \mid P_{1}$ regarded as a $C_{1}^{*}$-morphism into $[\Delta(A \hat{\otimes} B)]^{-}$. The fact that $\varkappa$ is surjective is a well-known simple consequence of Urysohn's lemma. Since $\pi^{*}\left(\Delta\left(A \otimes_{C} B\right)\right) \subset \Delta(A \hat{\otimes} B)$, it is quickly verified that $x$ is a semigroup homomorphism. Finally, $x\left(x_{1}, y_{1}\right)=\varkappa\left(x_{2}, y_{2}\right)$ for $x_{1}, x_{2} \in S_{A}, y_{1}, y_{2} \in S_{B}$ if and only if $\left(x_{1}, y_{1}\right)$ and $\left(x_{2}, y_{2}\right)$ determine the same functional on $[\Delta]^{-} C C\left(S_{A} \times S_{B}\right)$, i.e. if and only if $\varphi\left(x_{1}\right) \psi\left(y_{1}\right)=\varphi \otimes \psi\left(x_{1}, y_{1}\right)=$ $\varphi \otimes \psi\left(x_{2}, y_{2}\right)=\varphi\left(x_{2}\right) \psi\left(y_{2}\right)$ for all pairs $(\varphi, \psi)$ satisfying (i) to (v).

\section{Examples of CCM-actions}

Many natural actions of one commutative CM-algebra on another arising in harmonic analysis are CCM-actions. As the inclusion map of an $L$-subspace of an $L$-space $M$ into $M$ is an $L$-homomorphism (see the discussion preceding Lemma 4.2), it is clear from [19, Theorem 3.2] that a commutative CM-algebra is a CCM-module over any of its $L$-subalgebras. More generally, if $C$ and $A$ are commutative CM-algebras and $T: C \rightarrow A$ is an $L$-homomorphism which is also an algebra homomorphism, then the operator $\Theta: C \widehat{\otimes} A \rightarrow A$ for which $\Theta(c \otimes a)=(T c) a$ is a CCM-action. An equally obvious example of a CCM-module is an $L$-ideal of a commutative CM-algebra $A$ when it is regarded as a module over $A$. From Theorems 3.1 and 4.1 in [20] it follows, in particular, that if $A$ is a semi-simple commutative CM-algebra whose structure semigroup has an identity and whose multiplier algebra $M(A)$ is also a CM-algebra with respect to the order defined by the positive cone $\{T \in M(A) \mid T x \geq 0$ if $x \in A, x \geq 0\}$, then $A$ is a CCM-module over $M(A)$. 
The case of a so-called essential CCM-action of a commutative group algebra $L^{1}(K)$ (of the bounded Borel measures absolutely continuous with respect to the Haar measure of the locally compact abelian topological group $K$ ) on another such algebra $L^{1}(G)$ can be fairly easily analyzed. The result is Theorem 5.1. A CCM-action $\Theta: C \widehat{\otimes} A \rightarrow A$ is called essential, if the range of $\Theta$ is dense in $A$ (see [10, p. 453]). In this situation actually $\Theta(C \hat{\otimes} A)=A$, because the range of every $L$-homomorphism is closed (see [1, p. 492]). In case $C$ has a bounded approximate identity and $\Theta$ is essential, every $a \in A$ can even be expressed as $a=\Theta\left(c \otimes a^{\prime}\right)$ for some $c \in C, a^{\prime} \in A \quad[10$, Proposition 3.4].

The o r e m 5.1. Let $K$ and $G$ be locally compact abelian topological groups. A CCM-action $\Theta: L^{1}(K) \hat{\otimes} L^{1}(G) \rightarrow L^{1}(G)$ is essential if and only if there is a continuous homomorphism $\varphi: K \rightarrow G$ such that

$$
\Theta(\nu \otimes \mu)=\widetilde{\varphi}(v) * \mu,
$$

where $\widetilde{\varphi}: M(K) \rightarrow M(G)$ is the natural extension of $\varphi$ obtained by defining $\widetilde{\varphi}(v)(E)=v\left(\varphi^{-1}(E)\right)$ for all Borel sets $E \subset G$.

Proof. We identify the character group $\widehat{K}$ of $K$ with $\Delta\left(L^{1}(K)\right)$ by $\langle\nu, \gamma\rangle=\int_{K} \gamma(y) d v(y), \quad v \in L^{1}(K), \quad \gamma \in \widehat{K} \subset L^{\infty}(K)$, and $\widehat{G}$ with $\Delta\left(L^{1}(G)\right)$.

Let $t: \widehat{G} \rightarrow \widehat{K} \cup\{0\}$ be the adjoint map of the action $\Theta$ (Lemma 4.1). Suppose $\Theta$ is essential so that its transpose $\Theta^{*}$ is injective. Since $\Theta^{*} \gamma \neq 0$ for $\gamma \in \widehat{G}$, and $\Theta^{*} \gamma=t(\gamma) \otimes \gamma \quad($ Lemma 4.1), we have $t(\widehat{G}) \subset \widehat{K}$. Furthermore, $t \mid \widehat{G}$ is a group homomorphism. In fact, if $\quad \gamma_{1}, \gamma_{2} \in \widehat{G}$, then $t\left(\gamma_{1} \gamma_{2}\right) \otimes \gamma_{1} \gamma_{2}=\Theta^{*}\left(\gamma_{1} \gamma_{2}\right)=\Theta^{*}\left(\gamma_{1}\right) \Theta^{*}\left(\gamma_{2}\right)=$ $\left(t\left(\gamma_{1}\right) \otimes \gamma_{1}\right)\left(t\left(\gamma_{2}\right) \otimes \gamma_{2}\right)=t\left(\gamma_{1}\right) t\left(\gamma_{2}\right) \otimes \gamma_{1} \gamma_{2}$, and so $t\left(\gamma_{1} \gamma_{2}\right)=t\left(\gamma_{1}\right) t\left(\gamma_{2}\right)$, because $\gamma_{1} \gamma_{2} \neq 0$. As $t: \hat{G} \rightarrow \widehat{K}$ is continuous when $\widehat{G}$ (resp. $\widehat{K}$ ) is given the relative $\sigma\left(L^{\infty}(G), L^{1}(G)\right)$ (resp. $\sigma\left(L^{\infty}(K), L^{1}(K)\right)$ ) topology, the Pontryagin duality theorem yields a continuous homomorphism $\varphi: K \rightarrow G$ whose dual mapping $t$ is. To show that $\Theta$ is induced by $\varphi$ as claimed, it is sufficient to verify the indentity

(2) $\langle\tilde{\varphi}(v) * \mu, \gamma\rangle=\langle\Theta(\nu \otimes \mu), \gamma\rangle, \quad v \in L^{1}(K), \mu \in L^{1}(G), \gamma \in \Delta\left(L^{1}(G)\right)$.

With the interpretation $\gamma \in \widehat{G} \subset L^{\infty}(G)$ the definition of $\widetilde{\varphi}$ gives

$$
\begin{gathered}
\langle\tilde{\varphi}(v) * \mu, \gamma\rangle= \\
=\langle\mu, \gamma\rangle \int_{G} \gamma(x) d \widetilde{\varphi}(v)(x)=\langle\mu, \gamma\rangle \int_{K} \gamma \circ \varphi(y) d \nu(y) .
\end{gathered}
$$


For $\gamma \in \Delta\left(L^{1}(G)\right)$ this implies $\langle\widetilde{\varphi}(v) * \mu, \gamma\rangle=\langle\nu, t(\gamma)\rangle\langle\mu, \gamma\rangle$, which is equivalent to (2) by Lemma 4.1. Assume, conversely, that $\Theta$ is induced by a continuous homomorphism $\varphi: K \rightarrow G$ via (1). Let $\mathscr{U}$ be the neighborhood basis of zero in $K$, directed by the natural order opposite to inclusion. For each $U \in \mathscr{U}$ choose a positive measure $v_{U} \in L^{1}(K)$ such that $\int_{K} d v_{U}(y)=1$, and $\operatorname{supp}\left(v_{U}\right) \subset U$. Then

$$
\lim _{U} \Theta\left(v_{U} \otimes \mu\right)=\mu, \mu \in L^{1}(G) .
$$

In fact, given $\varepsilon>0$ there is a neighborhood $V$ of zero in $G$ such that $\left\|f_{u}-f\right\|_{1}<\varepsilon$ for all $u \in V$, where $f d x=d \mu$ and $f_{u}(x)=f(x-u)$ (see $\left[11\right.$, p. 3]). Let $U_{0} \in \mathscr{U}$ satisfy $\varphi\left(U_{0}\right) \subset V$. Then

$$
\begin{aligned}
\left\|\widetilde{\varphi}\left(v_{U}\right) * \mu-\mu\right\| & =\int_{G}\left|\int_{G}(f(x-u)-f(x)) d \widetilde{\varphi}\left(v_{U}\right)(u)\right| d x \leq \\
& \leq \int_{V}\left\|f_{u}-f\right\|_{1} d \widetilde{\varphi}\left(v_{U}\right)(u)<\varepsilon
\end{aligned}
$$

for all $U \in \mathscr{U}, U \subset U_{0}$. Thus (3) holds, and so $\Theta$ is essential.

\section{References}

[1] Brown, G.: On convolution measure algebras. - Proc. London Math. Soc. (3) $20,1970,490-506$.

[2] Dixmier, J.: Les algèbres d'opérateurs dans l'espace hilbertien (Algèbres de von Neumann). 2ième éd. - Cahiers scientifiques, Fas. XXV, Gauthier-Villars, Paris, 1969.

[3] Dixmrér, J.: Les C*algèbres et leurs représentations. 2ième éd. - Cahiers scientifiques, Fas. XXIX, Gauthier-Villars, Paris, 1969.

[4] Gerbaum, B. R.: Tensor products of Banach algebras. - Canad. J. Math. $11,1959,297-310$.

[5] HEwiTT, E., and K. A. Ross: Abstract harmonic analysis. Vol. I. - SpringerVerlag, Berlin-Göttingen-Heidelberg, 1963.

[6] KERLIN, J. E.: Tensor products of group algebras. - Trans. Amer. Math. Soc. $175,1973,1-36$.

[7] MCKIIIIGAN, S. A., and A. J. WHITE: Representations of $L$-algebras. - Proc. London Math. Soc. (3) 25, 1972, 655-674.

[8] Rennison, J. F.: Arens products and measure algebras. - J. London Math. Soc. 44, 1969, 369-377.

[9] RickarT, C. E.: General theory of Banach algebras. - D. Van Nostrand Company, Inc., Princeton-Toronto-London, 1960.

[10] RIEFFEI, M. A.: Induced Banach representations of Banach algebras and locally compact groups. - J. Functional Analysis 1, 1967, 443-491. 
[11] Rudin, W.: Fourier analysis on groups. - Interscience Publishers, New YorkLondon, 1962.

[12] SAKAI, S.: $C^{*}$-algebras and $W^{*}$-algebras. - Springer-Verlag, Berlin-Heidelberg-New York, 1971.

[13] Schat'Ten, R.: A theory of cross-spaces. - Annals of Mathematics Studies, No. 26, Princeton, 1950.

[14] Semadeni, Z.: Banach spaces of continuous functions. - Monografie Matematyczne, Tom 55, PWN-Polish Scientific Publishers, Warszawa, 1971.

[15] TAylor, J. L.: The structure of convolution measure algebras. - Trans. Amer. Math. Soc. $119,1965,150-166$.

[16] TAyroR, J. L.: Noncommutative convolution measure algebras. - Pacific J. Math. 31, 1969, 809-826.

[17] TAYLOR, J. L.: Measure algebras. - CBMS Regional conference series in mathematics No. 16, 1973.

[18] Tomryama, J.: Tensor products of commutative Banach algebras. - Tohoku Math. J. 12, 1960, $147-154$.

[19] Ylinen, K.: Tensor products of complex $L$-spaces and convolution measure algebras. - Ann. Acad. Sci. Fenn. Ser. A I 558, 1973, 1-11.

[20] YLINEN, K.: The multiplier algebra of a convolution measure algebra. Pacific J. Math. 51, 1974, 327-337.

University of Helsinki

Department of Mathematics

SF-00100 Helsinki 10

Finland

Received 19 September 1974 